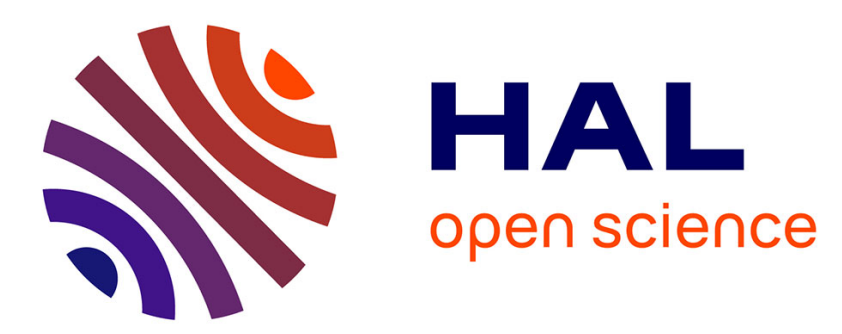

\title{
Continuous time quantum walk on an extended star graph: trapping and superradiance transition
}

\author{
Saad Yalouz, Vincent J.C. Pouthier
}

\section{To cite this version:}

Saad Yalouz, Vincent J.C. Pouthier. Continuous time quantum walk on an extended star graph: trapping and superradiance transition. Physical Review E , 2018, 97 (2), pp.022304. 10.1103/PhysRevE.97.022304 . hal-02294100

\section{HAL Id: hal-02294100 https://hal.science/hal-02294100}

Submitted on 23 Sep 2019

HAL is a multi-disciplinary open access archive for the deposit and dissemination of scientific research documents, whether they are published or not. The documents may come from teaching and research institutions in France or abroad, or from public or private research centers.
L'archive ouverte pluridisciplinaire HAL, est destinée au dépôt et à la diffusion de documents scientifiques de niveau recherche, publiés ou non, émanant des établissements d'enseignement et de recherche français ou étrangers, des laboratoires publics ou privés. 


\title{
Continuous time quantum walk on an extended star graph: trapping and superradiance transition.
}

\author{
Saad Yalouz and Vincent Pouthier* \\ Institut UTINAM, Univ. Bourgogne Franche-Comté, \\ CNRS UMR 6213, 25030 Besançon Cedex, France.
}

(Dated: January 23, 2018)

\begin{abstract}
A tight-binding model is introduced for describing the dynamics of an exciton on an extended star graph whose central node is occupied by a trap. On this graph, the exciton dynamics is governed by two kinds of eigenstates : many eigenstates are associated to degenerate real eigenvalues insensitive to the trap whereas three decaying eigenstates characterized by complex energies contribute to the trapping process. It is shown that the excitonic population absorbed by the trap depends on the size of the graph, only. By contrast, both the size parameters and the absorption rate control the dynamics of the trapping. When theses parameters are judiciously chosen, the efficiency of the transfer is optimized resulting in the minimization of the absorption time. The analysis of the eigenstates reveals that such a feature arises around the superradiance transition. Moreover, depending on the size of the network, two situations are highlighted where the transport efficiency is either super-optimized or sub-optimized.
\end{abstract}

PACS numbers:

\section{INTRODUCTION}

In large molecules and molecular crystals, understanding how excitons carry energy from one region to another is a key step for explaining many phenomena [1]. Examples among many concern Frenkel exciton in photosynthetic antenna [2] and J-aggregates [3], amide-I excitons in $\alpha$-helices [4] and vibrons in nanostructures [5]. During the last five decades, special attention has been paid for describing impurity induced exciton quenching in molecular lattices [6-10]. Indeed, a lattice involves regularly distributed donor ions which exchange excitations through various processes allowing the coherent or incoherent delocalization of excitons. In that case, an acceptor ion embedded in the lattice behaves as an irreversible trap because an exciton can be transferred to the acceptor whereas the reverse process has a negligible probability. Describing the exciton dynamics in the presence of traps is thus of fundamental importance for understanding many processes such as the time evolution of the fluorescence [11] and the exciton transport efficiency [12].

Although most studies were restricted to lattices with translational invariance [13-15], recent investigations were devoted to the characterization of the trapping phenomena in complex networks. Two kinds of systems were considered simultaneously.

On the one hand, the exciton trapping was investigated in realistic molecular networks with complex structures such as dendrimers [16] and Fenna-Matthews-Olson (FMO) protein [17]. Indeed, it has been shown that a dendrimer may behave as an artificial light-harvesting complex [18-23]. To proceed, the functionalization of the

\footnotetext{
*Electronic address: vincent.pouthier@univ-fcomte.fr
}

terminal groups by chromophores favors light harvesting. The capture of light generates excitons that converge toward the core that contains either a fluorescent trap, a reaction center, or a chemical sensor [24, 25]. Similarly, the FMO protein, which appears in green sulfur bacteria, is a pigment-protein complex that consists of seven bacteriochlorophyll-a molecules. In this protein, the absorption of light yields excitons able to propagate through the complex until they reach the reaction center where they are finally trapped [26-30].

On the other hand, trapping processes were studied from a more formal point of view by considering the complex networks one encounters in graph theory. Such studies result from the fact that the delocalization of an exciton between the nodes of a graph defines a continuous time quantum walk (CTQW) [31]. As the quantum analog of classical random walk, CTQW on complex networks appears to be a promising route to develop highperformance quantum algorithms [32, 33]. The trapping problem has been studied in a large variety of networks with a special emphasis on the comparison between CTQW and classical random walk. Examples among many are hyperbranched fractals [34], Sierpinsky fractals [35], cycle graphs with long-range interactions [36], chains and rings [37-39], and random networks [40].

From a theoretical point of view, the exciton trapping is usually addressed using an effective non-hermitian Hamiltonian approach [41, 42]. Within this approach, the trapping results from the coupling with an external continuum whose influence is encoded in an exciton Hamiltonian with complex eigenvalues. The real parts of these eigenvalues define the excitonic energies whereas the imaginary parts specify the energy widths (i.e. decay rates) resulting in an exciton finite lifetime. In that case, a detailed study of the complex excitonic eigenvalues has revealed the occurrence of a general phenomenon called superradiance transition (ST) [43]. The ST has 
been discovered in the context of quantum optic [44]. It is a cooperative phenomenon that occurs when $N$ excited emitters interact with a common electromagnetic field. If the wavelength of the light is greater than the separation of the emitters, a collective spontaneous emission arises. The emitters behave cooperatively resulting in a decay rate proportional to $N^{2}$ that is strongly enhanced when compared to the rate proportional to $\mathrm{N}$ observed when the emitters radiate independently of each other. However, after the works of Sokolov and Zelevinsky [45, 46], the ST has been recognized to be a general phenomenon that affects a quantum system opens to a continuum. Not restricted to many excitation effects, it can occur also in the presence of a single excitation, as encounter in the exciton trapping problem. Indeed, when the exciton is weakly coupled to a trap, all the exciton quantum states are similarly affected. They thus exhibit a quite similar energy width. However, as the exciton-trap coupling increases, a reconstitution of the eigenstates takes place. Only few short-lived states, called superradiant states, exhibit cooperatively enhanced decay rates. These states are accompanied by subradiant eigenstates, that is longlived states almost decoupled from the trap. As shown very recently, the ST has a very pronounced impact on the transport efficiency in complex networks, including formal graphs and realistic systems [47-49].

Drawing on these concepts, we consider in this paper the dynamics of an exciton moving on an extended star graph [50] whose central node is occupied by a trap. Special attention is paid for describing the ST and its consequence on the transport efficiency when the exciton propagates from the periphery to the core of the graph. Note that the choice of the extended star graph is not the result of chance. Indeed, this graph is one of the most regular structures in graph theory. Organized around a central core, it exhibits the local tree structure of irregular and complex networks. However, its topology remains sufficiently simple so that analytical calculations can be carried out resulting in a perfect understanding of the influence of the graph size on the transport properties. The present work can thus be viewed as a first step and more realistic situations will be addressed in forthcoming papers.

The paper is organized as follows. In Sec. II, the extended star graph is introduced and the exciton Hamiltonian is defined. Then, the exciton eigenstates and the relevant observables required for characterizing the dynamics are described. In Sec. III, a numerical analysis is performed for describing the trapping process. Finally, the results are discussed in Sec. IV.

\section{THEORETICAL BACKGROUND}

\section{A. Model Hamiltonian}

The system we consider is the extended star graph illustrated in Fig. 1a. It corresponds to a two-generation

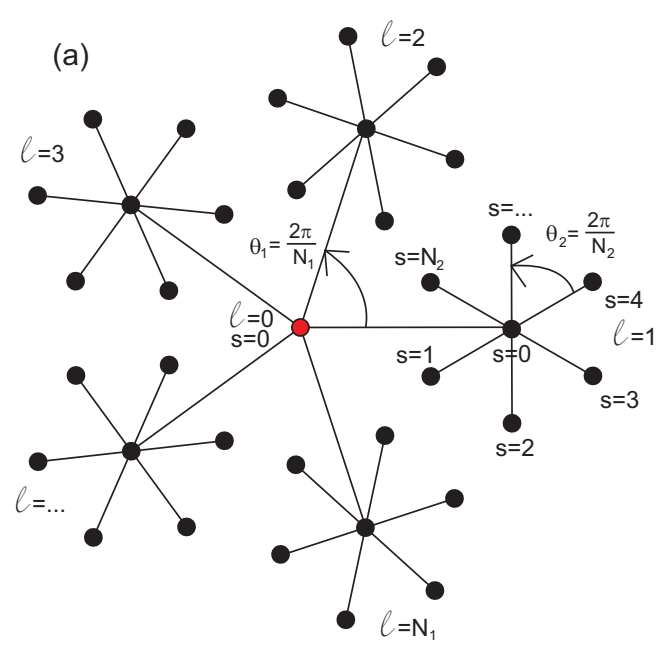

(b)

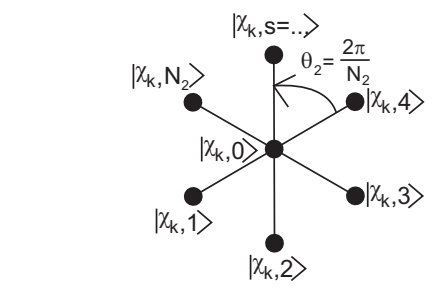

(c)

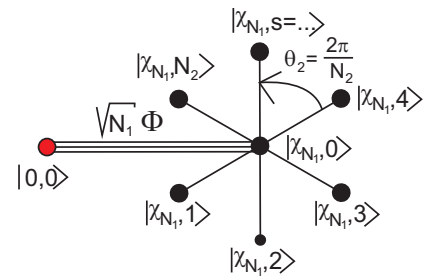

(d)

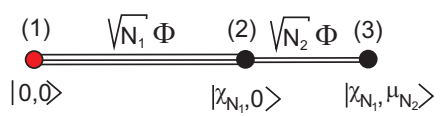

FIG. 1: (a) Representation of the extended star graph with $N=1+N_{1}\left(N_{2}+1\right)$ nodes $(\ell, s)$. Graphical representation of the block Hamiltonian (b) $H^{(k)}$ with $k \neq N_{1}$, (c) $H^{\left(N_{1}\right)}$ and (d) $\mathcal{H}$ (see the text).

dendrimer-like structure formed by $N_{1}$ branches that emanate out from a central core. Each branch connects the central core to the central node of $N_{1}$ peripheral star graphs. Each peripheral star graph involves $N_{2}$ branches so that the total number of sites is $N=1+N_{1}\left(N_{2}+1\right)$. For describing the graph, let us introduce the labels $(\ell, s)$ : $(\ell=0, s=0)$ refers to the central core, $(\ell, s=0)$, with $\ell=1, \ldots, N_{1}$, defines the central node of the $\ell$ th peripheral star and $(\ell, s)$, with $\ell=1, \ldots, N_{1}$ and $s=1, \ldots, N_{2}$, characterizes the $s$ th peripheral site of the $\ell$ th star.

The exciton dynamics is modeled as follows. Each site $(\ell, s)$ is occupied by a molecular subunit whose internal dynamics is described by a two-level system with Bohr frequency $\omega_{0}$ (using the Bohr frequency as the energy reference, the convention $\omega_{0}=0$ will be used in the following of the text). Let $|\ell, s\rangle$ denote the state in which the 
$(\ell, s)$ th two-level system occupies its first excited state, the other two-level systems remaining in their ground state. One assumes that a trap is located on the central core. This trap is responsible for an irreversible decay of the exciton according to the decay rate $\Gamma$. In that context, the exciton dynamics is governed by the nonhermitian effective Hamiltonian defined in terms of the hopping constant $\Phi$ as (within the convention $\hbar=1$ )

$$
\begin{aligned}
H & =(-i \Gamma / 2)|0,0\rangle\langle 0,0|+\sum_{\ell=1}^{N_{1}} \Phi(|0,0\rangle\langle\ell, 0|+\text { h.c. }) \\
& +\sum_{\ell=1}^{N_{1}} \sum_{s=1}^{N_{2}} \Phi(|\ell, 0\rangle\langle\ell, s|+\text { h.c. }),
\end{aligned}
$$

where h.c. stands for hermitian conjugate.

\section{B. System eigenstates}

For describing the exciton eigenstates, we take advantage of the fact that $H$ is invariant under the discrete rotation of angle $\theta_{1}=2 \pi / N_{1}$ and centered on the central core $(\ell=0, s=0)$. Consequently, its diagonalization is greatly simplified when one works with an intermediate Bloch basis that involves the local state $|0,0\rangle$ and $N_{1}\left(N_{2}+1\right)$ orthogonal Bloch states $\left|\chi_{k}, s\right\rangle\left(k=1, \ldots, N_{1}\right)$ defined as

$$
\left|\chi_{k}, s\right\rangle=\frac{1}{\sqrt{N_{1}}} \sum_{\ell=1}^{N_{1}} e^{i k \ell \theta_{1}}|\ell, s\rangle .
$$

Since $k$ is a good quantum number, the Hamiltonian $H$ becomes block diagonal. It is expressed as a direct sum $H=H^{(1)} \oplus H^{(2)} \oplus \ldots \oplus H^{\left(N_{1}\right)}$ where $H^{(k)}$ is the block Hamiltonian associated to the quantum number $k$.

For $k \neq N_{1}, H^{(k)}$ is a hermitian operator that acts in a subspace whose dimension is $N_{2}+1$, as

$$
H^{(k)}=\sum_{s=1}^{N_{2}} \Phi\left(\left|\chi_{k}, 0\right\rangle\left\langle\chi_{k}, s\right|+\text { h.c. }\right),
$$

$H^{(k)}$ corresponds to the Hamiltonian of an exciton moving on the star graph shown in Fig. 1b. This graph, whose sites are labeled by the index $s=0, \ldots, N_{2}$ (for each $k$ values), involves $N_{2}$ branches that connect the central site $s=0$ (i.e. the state $\left|\chi_{k}, 0\right\rangle$ ) to $N_{2}$ peripheral sites $s=1, \ldots, N_{2}$ (i.e. $N_{2}$ peripheral states $\left|\chi_{k}, s\right\rangle$ ). As a result, $H^{(k)}$ is invariant under the discrete rotation of angle $\theta_{2}=2 \pi / N_{2}$ and centered on the site $s=0$. Once again, its diagonalization is simplified by performing a Bloch transformation, that now runs over the index $s$. In doing so, one obtains a new basis involving the state $\left|\chi_{k}, 0\right\rangle$ and $N_{2}$ Bloch states $\left|\chi_{k}, \mu_{q}\right\rangle\left(q=1, \ldots, N_{2}\right)$ defined as

$$
\left|\chi_{k}, \mu_{q}\right\rangle=\frac{1}{\sqrt{N_{2}}} \sum_{s=1}^{N_{2}} e^{i q s \theta_{2}}\left|\chi_{k}, s\right\rangle .
$$

Within this basis, $H^{(k)}$ exhibits two kinds of eigenstates. First, its spectrum shows $\left(N_{2}-1\right)$-fold degenerate eigenenergy $\omega^{(k, q)}=0$, with $q=1, \ldots, N_{2}-1$, the corresponding eigenstates being the $N_{2}-1$ Bloch states $\left|\chi_{k}, \mu_{q}\right\rangle$. Second, $H^{(k)}$ supports two eigenstates $\left|\chi_{k}, \pm\right\rangle$ defined as

$$
\left|\chi_{k}, \pm\right\rangle=\frac{1}{\sqrt{2}}\left(\left|\chi_{k}, 0\right\rangle \pm\left|\chi_{k}, \mu_{N_{2}}\right\rangle\right),
$$

with eigenenergies $\omega_{ \pm}^{\left(k, N_{2}\right)}= \pm \sqrt{N_{2}} \Phi$.

For $k=N_{1}$, the block Hamiltonian $H^{\left(N_{1}\right)}$, whose size reduces to $N_{2}+2$, is defined as

$$
\begin{aligned}
H^{\left(N_{1}\right)} & =-i \Gamma / 2|0,0\rangle\langle 0,0|+\sqrt{N_{1}} \Phi\left(|0,0\rangle\left\langle\chi_{N_{1}}, 0\right|+\text { h.c. }\right) \\
& +\sum_{s=1}^{N_{2}} \Phi\left(\left|\chi_{N_{1}}, 0\right\rangle\left\langle\chi_{N_{1}}, s\right|+\text { h.c. }\right) .
\end{aligned}
$$

$H^{\left(N_{1}\right)}$ is equivalent to the Hamiltonian of an exciton moving on the graph displayed in Fig. 1c. This graph exhibits $\mathrm{N}_{2}+2$ sites organized as follows. The first site is associated to the state $|0,0\rangle$ that is localized on the central core of the extended star. It is connected to the second site associated to the state $\left|\chi_{N_{1}}, 0\right\rangle$ through the hopping constant $\sqrt{N_{1}} \Phi$. The second site is the center of a star graph that exhibits $N_{2}$ branches. As previously, $H^{\left(N_{1}\right)}$ is invariant under the discrete rotation of angle $\theta_{2}=2 \pi / N_{2}$ so that its diagonalization is simplified by introducing a new basis involving the state $|0,0\rangle$, the state $\left|\chi_{N_{1}}, 0\right\rangle$ and $N_{2}$ Bloch states $\left|\chi_{N_{1}}, \mu_{q}\right\rangle\left(q=1, \ldots, N_{2}\right)$ (see Eq.(4)).

Within this basis, $H^{\left(N_{1}\right)}$ exhibits $\left(N_{2}-1\right)$ degenerate eigenstates $\left|\chi_{N_{1}}, \mu_{q}\right\rangle$, with $q=1, \ldots, N_{2}-1$, whose eigenenergy is equal to $\omega^{\left(N_{1}, q\right)}=0$. Therefore, the three remaining eigenstates are those of the $(3 \times 3)$ nonhermitian matrix that corresponds to the restriction of the block $H^{\left(N_{1}\right)}$ to the subspace generated by the three vectors $|0,0\rangle,\left|\chi_{N_{1}}, 0\right\rangle$ and $\left|\chi_{N_{1}}, \mu_{N_{2}}\right\rangle$. This restriction, denoted $\mathcal{H}$, defines the Hamiltonian of an exciton moving on the trimer shown in Fig. 1d and whose sites 1, 2 and 3 are associated to the states $|0,0\rangle,\left|\chi_{N_{1}}, 0\right\rangle$ and $\left|\chi_{N_{1}}, \mu_{N_{2}}\right\rangle$, respectively. $\mathcal{H}$ is expressed as

$$
\mathcal{H}=\left(\begin{array}{ccc}
-i \Gamma / 2 & \sqrt{N_{1}} \Phi & 0 \\
\sqrt{N_{1}} \Phi & 0 & \sqrt{N_{2}} \Phi \\
0 & \sqrt{N_{2}} \Phi & 0
\end{array}\right)
$$

When $\Gamma=0, \mathcal{H}$ is a hermitian operator that can be diagonalized exactly. It exhibits three eigenvectors, as

$$
\begin{aligned}
\left|\Psi_{ \pm}\right\rangle & =\frac{|0,0\rangle \pm \sqrt{1+r}\left|\chi_{N_{1}}, 0\right\rangle+\sqrt{r}\left|\chi_{N_{1}}, \mu_{N_{2}}\right\rangle}{\sqrt{2(1+r)}} \\
\left|\Psi_{0}\right\rangle & =\frac{\sqrt{r}|0,0\rangle-\left|\chi_{N_{1}}, \mu_{N_{2}}\right\rangle}{\sqrt{1+r}}
\end{aligned}
$$

with $r=N_{2} / N_{1}$. The corresponding eigenvalues are $\hat{\omega}_{ \pm}^{\left(N_{1}, N_{2}\right)}= \pm \sqrt{N_{1}+N_{2}} \Phi$ and $\hat{\omega}_{0}^{\left(N_{1}, N_{2}\right)}=0$. When $\Gamma \neq 0, \mathcal{H}$ is diagonalized numerically. Due to its 
non-hermiticity, it exhibits three eigenvalues that are complex numbers [51] whose real parts define effective energies and whose imaginary parts define decay rates (i.e. widths). These eigenvalues are denoted $\hat{\omega}_{ \pm}^{\left(N_{1}, N_{2}\right)}=\omega_{ \pm}^{\left(N_{1}, N_{2}\right)}-i \gamma_{ \pm}^{\left(N_{1}, N_{2}\right)} / 2$ and $\hat{\omega}_{0}^{\left(N_{1}, N_{2}\right)}=$ $\omega_{0}^{\left(N_{1}, N_{2}\right)}-i \gamma_{0}^{\left(N_{1}, N_{2}\right)} / 2$. These eigenvalues are associated to right eigenvectors $\left|\Psi_{ \pm}\right\rangle$and $\left|\Psi_{0}\right\rangle$ and to left eigenvectors $\left|\tilde{\Psi}_{ \pm}\right\rangle$and $\left|\tilde{\Psi}_{0}\right\rangle$. Note that these eigenvectors will be chosen to be biorthonormal $\left(\left\langle\tilde{\Psi}_{i} \mid \Psi_{j}\right\rangle=\delta_{i, j}\right)$ and to satisfy the completeness relation $\sum_{i}\left|\Psi_{i}\right\rangle\left\langle\tilde{\Psi}_{i}\right|=I_{t}$, where $I_{t}$ is the trimer subspace identity operator.

To summarize, the eigenstates can be divided into two main types. First, the exciton Hamiltonian supports $N_{1}\left(N_{2}+1\right)-2$ eigenstates associated to real eigenvalues. These states, denoted $\left|\chi_{k}, \mu_{q}\right\rangle$ with $k=1, \ldots, N_{1} ; q=$ $1, \ldots, N_{2}$ and $\left|\chi_{k}, \pm\right\rangle$ with $k=1, \ldots, N_{1}-1$, are insensitive to the presence of the trap. Then, the three remaining eigenstates are those of the restriction of the Hamiltonian to the trimer subspace. The trimer subspace is generated by three states : $|1\rangle=|0,0\rangle$ (located at the center of the extended star graph), $|2\rangle=\left|\chi_{N_{1}}, 0\right\rangle$ (uniformly distributed over the center of the peripheral star) and $|3\rangle=\left|\chi_{N_{1}}, \mu_{N_{2}}\right\rangle$ (uniformly distributed over the peripheral sites). Because the trap is located at the center of the graph, the corresponding eigenstates define decaying states characterized by complex energies.

\section{Quantum dynamics}

In the present study, we consider that the exciton is initially located on a peripheral site $\left(\ell_{0}=1, s_{0}=1\right)$ of the extended star graph. Its quantum state at time $t=0$ is thus defined as $|\Psi(0)\rangle=\left|\ell_{0}, s_{0}\right\rangle$. In that context, the exciton quantum state at time $t$ is obtained by solving the standard time dependent Schrodinger equation. A formal solution is encoded in the evolution operator $U(t)=\exp (-i H t)$ that satisfies $|\Psi(t)\rangle=U(t)|\Psi(0)\rangle$. From the knowledge of the exciton eigenstates, the evolution operator can be determined easily, as

$$
\begin{aligned}
U(t) & =\sum_{k=1}^{N_{1}} \sum_{q=1}^{N_{2}-1}\left|\chi_{k}, \mu_{q}\right\rangle\left\langle\chi_{k}, \mu_{q}\right| e^{-i \omega^{(k, q)} t} \\
& +\sum_{k=1}^{N_{1}-1} \sum_{\sigma= \pm}\left|\chi_{k}, \sigma\right\rangle\left\langle\chi_{k}, \sigma\right| e^{-i \omega_{\sigma}^{\left(k, N_{2}\right)} t} \\
& +\sum_{i=0, \pm}\left|\Psi_{i}\right\rangle\left\langle\tilde{\Psi}_{i}\right| e^{-i \hat{\omega}_{i}^{\left(N_{1}, N_{2}\right)} t} .
\end{aligned}
$$

The characterization of both the evolution operator and the eigenstates, allows us to compute different observables. First, we shall focus our attention on the exciton density $P_{\ell, s \mid \ell_{0}, s_{0}}(t)$ that represents the probability to observe the exciton on a site $(\ell, s)$ at time $t$, as

$$
P_{\ell, s \mid \ell_{0}, s_{0}}(t)=\left|\left\langle\ell, s|U(t)| \ell_{0}, s_{0}\right\rangle\right|^{2} .
$$

Then, two quantities are of particular interest for describing the exciton dynamics in the presence of a trap. The survival probability $Q(t)$ defines the probability that the exciton can still be found on the graph after some time $t$. The absorbed population $P_{A}(t)$ is the probability that the exciton is absorbed by the trap at time $t$. Both quantities are defined as

$$
\begin{aligned}
Q(t) & =\sum_{\ell, s} P_{\ell, s \mid \ell_{0}, s_{0}}(t) \\
P_{A}(t) & =1-Q(t) .
\end{aligned}
$$

Finally, the efficiency of the exciton transport is measured by the total absorbed population $P_{A}(+\infty)$ [49], and by the absorption time $\tau$, i.e. the time for which the absorbed population reaches $99 \%$ of its maximum value.

Note that, when $\Gamma=0, P_{\ell, s \mid \ell_{0}, s_{0}}(t)$ does not converge to a stationary value because a unitary dynamics arises [52]. Instead, it fluctuates around a long time average distribution called the limiting probability $\bar{P}_{\ell, s \mid \ell_{0}, s_{0}}$. It is defined as

$$
\bar{P}_{\ell, s \mid \ell_{0}, s_{0}}=\lim _{T \rightarrow \infty} \frac{1}{T} \int_{0}^{T} P_{\ell, s \mid \ell_{0}, s_{0}}(t) d t .
$$

The exciton density, the absorbed population, the limiting probability and the absorption time are the central objects of the present study. They give information about the way the exciton propagates along the network after its initial implementation. Their knowledge allows us to characterize the influence of the trap, as illustrated in the next sections.

\section{NUMERICAL RESULTS}

\section{A. Coherent Exciton transport : $\Gamma=0$}

Before considering the influence of the trap, let us first summarize what happens when the exciton moves coherently on the extended star. In that case, since we are able to diagonalize the system Hamiltonian, an exact expression of the exciton density is obtained, as illustrated in appendix A. Therefore, a measure of the transport efficiency is given by the probability to observe the exciton on the central node at time $t$ defined as

$$
P_{0,0 \mid \ell_{0}, s_{0}}(t)=\left(\frac{2}{N_{1}+N_{2}}\right)^{2} \sin ^{4}\left(\frac{\sqrt{N_{1}+N_{2}} \Phi t}{2}\right) .
$$

This probability is a periodic function whose period is equal to $T=2 \pi / \sqrt{N_{1}+N_{2}} \Phi$. It varies around $\bar{P}_{0,0 \mid \ell_{0}, s_{0}}=1.5 /\left(N_{1}+N_{2}\right)^{2}$ and it reaches a maximum value $P_{0,0 \mid \ell_{0}, s_{0}}^{\max }=4 /\left(N_{1}+N_{2}\right)^{2}$. The size parameters $N_{1}$ and $N_{2}$ affect similarly the behavior of the probability so that the larger the lattice size is, the smaller is the probability that the exciton reaches the core of the graph. For instance, with $N_{1}=N_{2}=3$, one obtains $P_{0,0 \mid \ell_{0}, s_{0}}^{\max }=0.11$. 

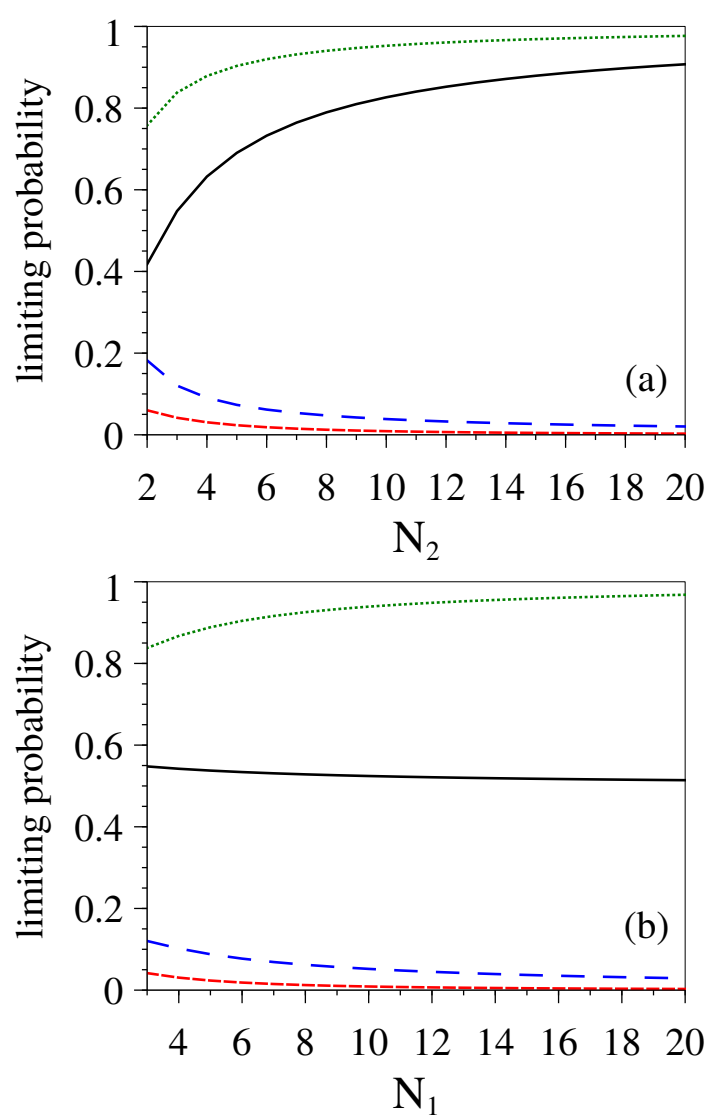

FIG. 2: (Color online) (a) $N_{2}$ dependence of the limiting probability for $N_{1}=3$ and (b) $N_{1}$ dependence of the limiting probability for $N_{2}=3$. Limiting probabilities to observe the exciton on the excited site (black full line), on the excited star (green dotted line), on the other stars (blue dashed line) and on the central core (red short dashed line).

To understand this feature, let us discuss the behavior of the limiting probabilities shown in Fig. 2. As illustrated in Fig. 2a for $N_{1}=3$, the limiting probabilities to observe the exciton on the excited site (black full line) and on the excited star (green dotted line) increase as $N_{2}$ increases. The larger $N_{2}$ is, the closer are the two curves. By contrast, the limiting probability to observe the exciton on the other stars decreases with $N_{2}$ (blue dashed line). In other words, increasing the size $N_{2}$ of the peripheral stars favors a localization of the exciton on the excited site. As shown in Fig. 2b, a different behavior arises when the size $N_{1}$ varies. Indeed, for $N_{2}=3$, the limiting probability to observe the exciton on the excited site (black full line) is almost $N_{1}$ independent. It slightly decreases with $N_{1}$ and it varies from 0.55 for $N_{1}=2$ to 0.5 for $N_{1}=20$. Conversely, the limiting probability to observe the exciton on the excited star (green dotted line) increases as $N_{1}$ increases whereas the limiting probability to observe the exciton on the other stars decreases with $N_{1}$ (blue dashed line). In that case, it turns out that increasing the size $N_{1}$ yields a localization on the excited star. Nevertheless, inside that excited star, the excitonic distribution is mainly governed by the $N_{2}$ parameter, as observed in Fig. 2a.

According to Eq.(9), the probability amplitude to observe the exciton on the central core is the sum of the probability amplitudes associated to the different paths that the exciton can follow to tunnel from the periphery to the core. A path defines a transition through a specific eigenstate so that two kinds of contributions arise. The first contribution corresponds to paths involving the eigenstates of the restriction of the Hamiltonian to the trimer subspace. Because of the extended nature of these states, this contribution favors the delocalization of the exciton. By contrast, the second contribution involves the sum of the probability amplitudes associated to degenerate eigenstates. This sum gives rise to quantum interferences that favor the localization of the exciton over few sites that surround the excited site. As the size of the network increases, the second contribution dominates preventing the exciton to reach the core of the graph. Such an effect, well-known in complex networks, corresponds to the process of localization induced by degeneracy $[53,54]$.

\section{B. Influence of the trap : $\Gamma \neq 0$}

When the presence of the trap is taken into account, the part of the exciton population that reaches the central core is now absorbed. The time evolution of the absorbed population is shown in Fig. 3 for different values of the size parameters $N_{1}$ and $N_{2}$. For $\Gamma=3 \Phi$ (Fig. 3a), $P_{A}(t)$ first scales as $t^{5}$ in the very short-time limit, whatever the value of the size parameters. Then, it increases as time increases and, in the long-time limit, it converges to a constant value $P_{A}(\infty)$. An important fact is that $P_{A}(\infty)$ clearly depends on the size parameters but it is invariant under the exchange of the latter. For $\left(N_{1}=3, N_{2}=8\right)$ and $\left(N_{1}=8, N_{2}=3\right)$, it reaches $P_{A}(\infty)=0.041$, whereas for $\left(N_{1}=3, N_{2}=6\right)$ and $\left(N_{1}=6, N_{2}=3\right)$, it is equal to $P_{A}(\infty)=0.055$. This behavior has been verified in several simulations in which we intentionally inverted the values of $N_{1}$ and $N_{2}$. To reach this asymptotic limit, $P_{A}(t)$ follows an exponential function that rises to a maximum modulated by a series of plateaus. The number of plateaus and their duration depend on the size parameters. Consequently, for the same absorbed population, the absorption time differs from $\tau=9.72 \Phi^{-1}$ for $\left(N_{1}=3, N_{2}=6\right)$ to $\tau=5.06 \Phi^{-1}$ for $\left(N_{1}=6, N_{2}=3\right)$. Similarly, for $\left(N_{1}=3, N_{2}=8\right)$ the absorption time is $\tau=11.95 \Phi^{-1}$ whereas for $\left(N_{1}=8, N_{2}=3\right)$ it reduces to $\tau=5.16 \Phi^{-1}$.

When $\Gamma=20 \Phi$ (Fig. 3b), a similar behavior is observed, but over different timescales. Indeed, $P_{A}(\infty)$ is clearly $\Gamma$ independent so that we recover the asymptotic values obtained in Fig. 3a. However the time needed to reach $P_{A}(\infty)$ is now more important. One obtains $\tau=15.97 \Phi^{-1}$ for $\left(N_{1}=3, N_{2}=6\right)$ and $\tau=7.86 \Phi^{-1}$ for 


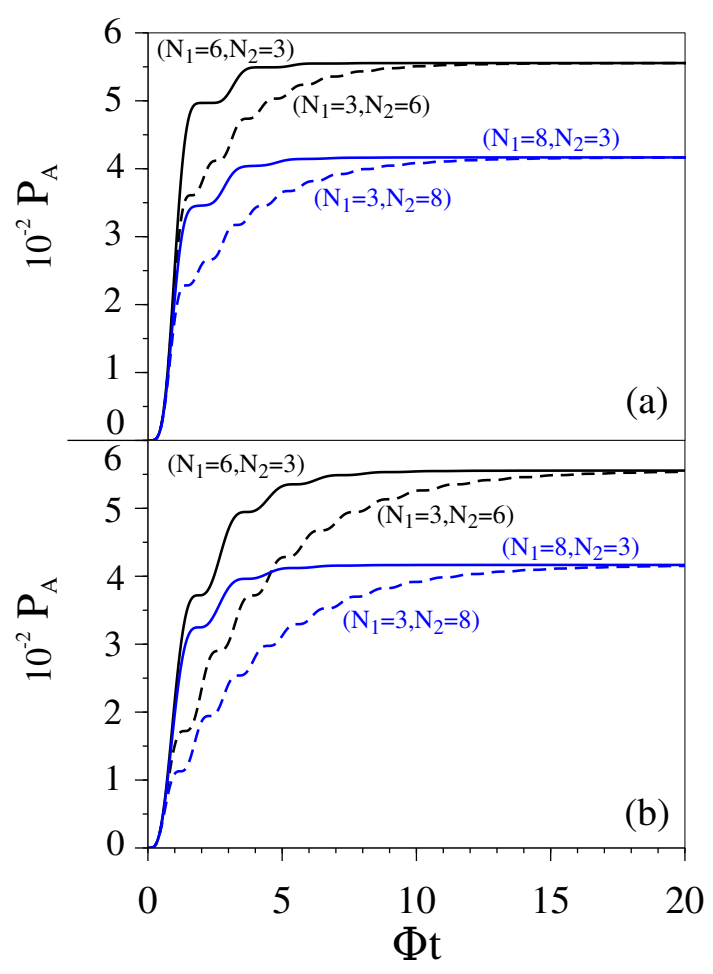

FIG. 3: (Color online) Time evolution of the absorbed population $P_{A}(t)$ for (a) $\Gamma=3 \Phi$ and for (b) $\Gamma=20 \Phi$. $\left(N_{1}=8, N_{2}=3\right)$ blue full line, $\left(N_{1}=3, N_{2}=8\right)$ blue dashed line, $\left(N_{1}=6, N_{2}=3\right)$ black full line,

$\left(N_{1}=3, N_{2}=6\right)$ black dashed line.

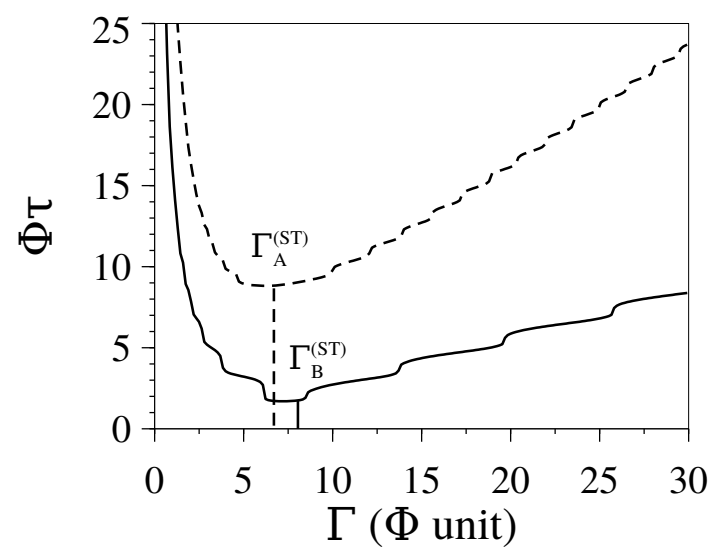

FIG. 4: $\Gamma$ dependence of the absorption time $\tau$. Configuration A : $\left(N_{1}=3, N_{2}=8\right)$ dashed line. Configuration B : $\left(N_{1}=8, N_{2}=3\right)$ full line.

$\left(N_{1}=6, N_{2}=3\right)$. Similarly, for $\left(N_{1}=3, N_{2}=8\right)$ the absorption time is $\tau=16.16 \Phi^{-1}$ whereas for $\left(N_{1}=8, N_{2}=\right.$ 3 ) it reduces to $\tau=5.88 \Phi^{-1}$. In fact, these observations are quite general and we have verified that the parameter $\Gamma$ does not affect the total absorbed population. It governs only the dynamics of the trapping process and it controls the value of the absorption time $\tau$.

To illustrate these features, the $\Gamma$ dependence of the

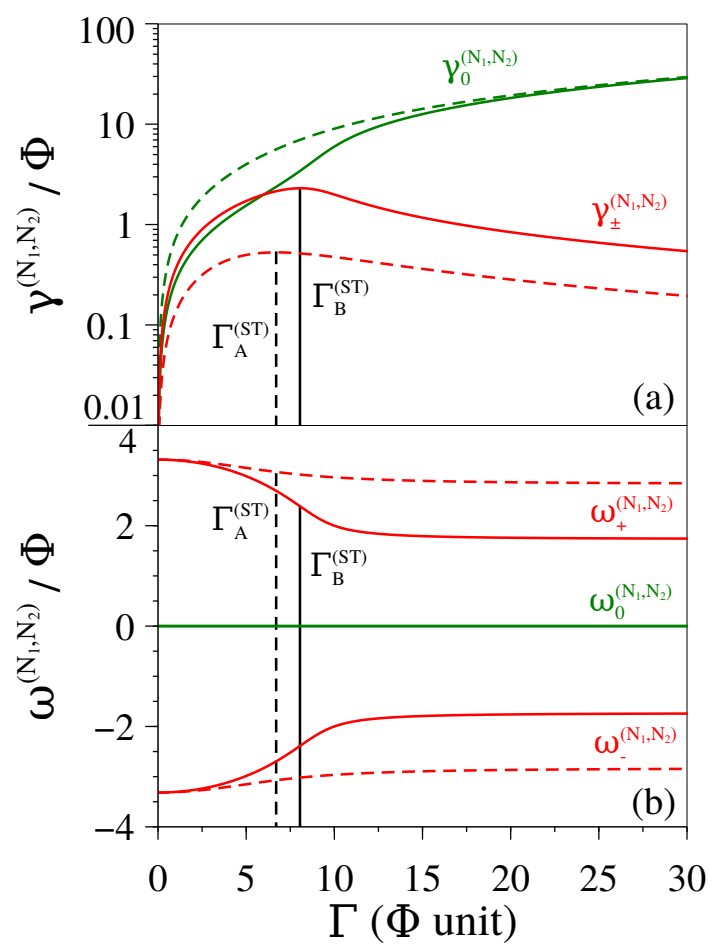

FIG. 5: (Color online) $\Gamma$ dependence of (a) the width and (b) the energy of the decaying states for configurations A (dashed lines) and B (full lines): $\gamma_{0}^{\left(N_{1}, N_{2}\right)}$ and $\omega_{0}^{\left(N_{1}, N_{2}\right)}$ (green curve); $\gamma_{ \pm}^{\left(N_{1}, N_{2}\right)}$ and $\omega_{ \pm}^{\left(N_{1}, N_{2}\right)}$ (red curve).

absorption time $\tau$ is shown in Fig. 4. To proceed, two configurations were considered. The configuration denoted A corresponds to $\left(N_{1}=3, N_{2}=8\right)$ whereas the configuration denoted B refers to $\left(N_{1}=8, N_{2}=3\right)$. Although in both cases the total absorbed population is the same $\left(P_{A}(\infty)=0.041\right)$, a different dynamics occurs.

For the configuration A, a quite long absorption time arises for small $\Gamma$ values. For instance, $\tau=20 \Phi^{-1}$ for $\Gamma=1.7 \Phi$. However, $\tau$ decreases rapidly as $\Gamma$ increases. It reaches a minimum value $\tau=8.80 \Phi^{-1}$ when $\Gamma \in[4.8 \Phi, 9.8 \Phi]$. This interval defines the $\Gamma$ range for which the transport efficiency is optimized. Then, as $\Gamma$ increases, $\tau$ slightly increases and one recovers $\tau=20 \Phi^{-1}$ for $\Gamma=25 \Phi$. Note that the curve $\tau$ vs $\Gamma$ is slightly modulated by weakly pronounced plateaus. For the configuration B, a similar behavior takes place. Indeed, $\tau$ first decreases as $\Gamma$ increases from zero. Then, it reaches a minimum value and finally it increases with $\Gamma$. The curve $\tau$ vs $\Gamma$ is still modulated by plateaus which are now more pronounced. Nevertheless, a difference clearly occurs : the minimum value of the absorption time reduces to $\tau=1.77 \Phi^{-1}$ indicating that the optimization of the transport efficiency is enhanced by the exchange of the size parameters. This optimization occurs when $\Gamma \in[6.2 \Phi, 8.5 \Phi]$.

To understand the physics behind the $\Gamma$-induced opti- 
mization of the transport efficiency, as well as the influence of the size parameters, let us study the $\Gamma$ dependence of the decaying eigenstates, i.e. the only states that contribute to the absorption process. The exciton supports three decaying eigenstates denoted $\left|\Psi_{0}\right\rangle$ and $\left|\Psi_{ \pm}\right\rangle$(see Sec.II).

In Fig. 5a, the $\Gamma$ dependence of the width of the decaying states is shown. For configuration $A$, the width $\gamma_{0}^{\left(N_{1}, N_{2}\right)}$ of the state $\left|\Psi_{0}\right\rangle$ (green dashed line) is a monotonous function that increases as $\Gamma$ increases. By contrast, the width $\gamma_{ \pm}^{\left(N_{1}, N_{2}\right)}$ of the states $\left|\Psi_{ \pm}\right\rangle$(red dashed line) behaves differently. For small $\Gamma$ values, quite close to $\gamma_{0}^{\left(N_{1}, N_{2}\right)}, \gamma_{ \pm}^{\left(N_{1}, N_{2}\right)}$ increases as $\Gamma$ increases. However, it reaches a maximum value $\gamma_{ \pm}^{\left(N_{1}, N_{2}\right)}=0.53 \Phi$ when $\Gamma=6.70 \Phi$. Then, it decreases as $\Gamma$ increases and becomes extremely small for large $\Gamma$ values. In fact, as explained by Zhang et al. [49], the non monotonous behavior of $\gamma_{ \pm}^{\left(N_{1}, N_{2}\right)}$ is the signature of the ST. The state $\left|\Psi_{0}\right\rangle$ defines the superradiant state whose lifetime decreases as $\Gamma$ increases. The two states $\left|\Psi_{ \pm}\right\rangle$correspond to subradiant states that become almost insensitive to the trap for large $\Gamma$ values. The ST occurs when $\Gamma=\Gamma_{A}^{(S T)}=6.70 \Phi$ $\left(\gamma_{0}^{\left(N_{1}, N_{2}\right)}=5.61 \Phi\right.$ at ST). For configuration B, the same features are observed. The width $\gamma_{0}^{\left(N_{1}, N_{2}\right)}$ (green full line) of the superradiant state increases monotonously as $\Gamma$ increases. By contrast, the width $\gamma_{ \pm}^{\left(N_{1}, N_{2}\right)}$ (red full line) of the subradiant states behaves non monotonously and it reaches a maximum $\gamma_{ \pm}^{\left(N_{1}, N_{2}\right)}=2.31 \Phi$ for $\Gamma=$ $\Gamma_{B}^{(S T)}=8.01 \Phi\left(\gamma_{0}^{\left(N_{1}, N_{2}\right)}=3.40 \Phi\right.$ at ST $)$. At this step, let us mention that $\Gamma_{A}^{(S T)}$ (resp. $\Gamma_{B}^{(S T)}$ ) belongs to the $\Gamma$ range where the optimization of the transport efficiency occurs for configuration A (resp. configuration B).

The $\Gamma$ dependence of the energy of the decaying states is illustrated in Fig. 5b for configurations A (dashed line) and $B$ (full line). Whatever the configuration, the energy of the superradiant state is $\Gamma$ independent. It is equal to $\omega_{0}=0$, i.e the eigenenergy of the state $\left|\Psi_{0}\right\rangle$ without the trap. A different behavior arises for the subradiant states. Equal to $\omega_{ \pm}^{\left(N_{1}, N_{2}\right)}= \pm \sqrt{N_{1}+N_{2}} \Phi$ for $\Gamma=0$, the energy of the subradiant states get closer to each other as $\Gamma$ increases. Such an effect arises around the ST. For the configuration A, the relevant Bohr frequency $\delta \omega=\omega_{+}^{\left(N_{1}, N_{2}\right)}-\omega_{-}^{\left(N_{1}, N_{2}\right)}$ is equal to $\delta \omega_{A}=6.14 \Phi$ at the ST $\left(\Gamma=\Gamma_{A}^{(S T)}\right)$. By contrast, for the configuration $\mathrm{B}$, it reduces $\delta \omega_{B}=4.77 \Phi$ for $\Gamma=\Gamma_{B}^{(S T)}$. Then, for larger $\Gamma$ values, we have verified that the energy tends to $\omega_{ \pm}^{\left(N_{1}, N_{2}\right)}= \pm \sqrt{N_{2}} \Phi$.

The $\Gamma$ dependence of the decaying states is illustrated in Fig. 6 for the configurations A (Figs. 6a and 6b) and B (Figs. 6c and 6d). The figures show the weight $\Pi_{n}$ of each decaying state on $|0,0\rangle\left(n=1\right.$, full line), $\left|\chi_{N_{1}}, 0\right\rangle$ $\left(n=2\right.$, dashed line) and $\left|\chi_{N_{1}}, \mu_{N_{2}}\right\rangle(n=3$, dotted line).

As shown in Fig. 6a, For configuration $\mathrm{A}$, when $\Gamma=0$, the superradiant state is an asymmetric superposition of the states $|0,0\rangle$ and $\left|\chi_{N_{1}}, \mu_{N_{2}}\right\rangle$ in which the weight of

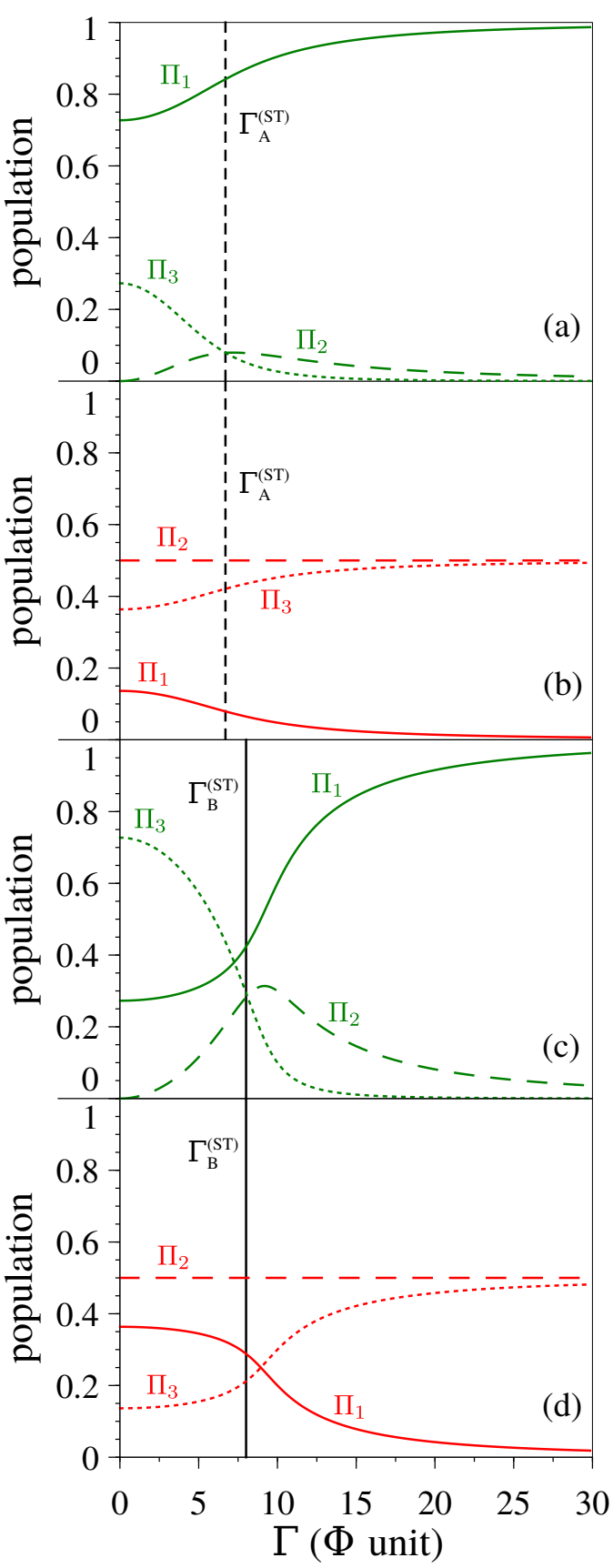

FIG. 6: (Color online) $\Gamma$ dependence for configuration $A$ of (a) the superradiant state and (b) the subradiant states; $\Gamma$ dependence for configuration $B$ of $(c)$ the superradiant state and (d) the subradiant states. The figures show the weight $\Pi_{n}$ of each decaying state on $|0,0\rangle$ ( $n=1$, full line), $\left|\chi_{N_{1}}, 0\right\rangle(n=2$, dashed line) and $\left|\chi_{N_{1}}, \mu_{N_{2}}\right\rangle(n=3$, dotted line $)$.

$|0,0\rangle$ is larger than the weight of $\left|\chi_{N_{1}}, \mu_{N_{2}}\right\rangle$. As $\Gamma$ increases, the weight of the state $|0,0\rangle$ increases whereas the weight of the state $\left|\chi_{N_{1}}, \mu_{N_{2}}\right\rangle$ decreases. Therefore, the weight of the state $\left|\chi_{N_{1}}, 0\right\rangle$ switches on. It increases with $\Gamma$ until it reaches a maximum value near the ST $\left(\Gamma \approx \Gamma_{A}^{S T}\right)$. In that case, the weights of $\left|\chi_{N_{1}}, \mu_{N_{2}}\right\rangle$ 
and $\left|\chi_{N_{1}}, 0\right\rangle$ are almost identical. Finally, for large $\Gamma$ values, the weights of $\left|\chi_{N_{1}}, \mu_{N_{2}}\right\rangle$ and $\left|\chi_{N_{1}}, 0\right\rangle$ decreases with $\Gamma$. They tend to zero indicating that the superradiant state becomes $|0,0\rangle$, i.e. it localized on the central core. As illustrated in Fig. 6b, when $\Gamma=0$, the subradiant states are superpositions delocalized over the three states $|0,0\rangle,\left|\chi_{N_{1}}, 0\right\rangle$, and $\left|\chi_{N_{1}}, \mu_{N_{2}}\right\rangle$. As $\Gamma$ increases, the weight of the state $|0,0\rangle$ decreases whereas the weight of the state $\left|\chi_{N_{1}}, \mu_{N_{2}}\right\rangle$ increases. By contrast, the weight of the state $\left|\chi_{N_{1}}, 0\right\rangle$ is equal to $0.5 \forall \Gamma$. As a consequence, for large $\Gamma$ values, the weight of $|0,0\rangle$ tends to zero so that the subradiant states become superpositions $\left(\left|\chi_{N_{1}}, 0\right\rangle \pm\left|\chi_{N_{1}}, \mu_{N_{2}}\right\rangle\right) / \sqrt{2}$.

For configuration $\mathrm{B}$, similar features are observed. In particular, for large $\Gamma$ values, one recovers that the superradiant state localizes on the central core (Fig. 6c) whereas the subradiant states become superpositions $\left(\left|\chi_{N_{1}}, 0\right\rangle \pm\left|\chi_{N_{1}}, \mu_{N_{2}}\right\rangle\right) / \sqrt{2}$ (Fig. 6d). In fact, the main difference with configuration $\mathrm{A}$ is the structure of the states for small $\Gamma$ values. As shown in Fig. $6 \mathrm{c}$, when $\Gamma=0$, the superradiant state is still an asymmetric superposition of the states $|0,0\rangle$ and $\left|\chi_{N_{1}}, \mu_{N_{2}}\right\rangle$. But now the weight of $|0,0\rangle$ is smaller than the weight of $\left|\chi_{N_{1}}, \mu_{N_{2}}\right\rangle$. As $\Gamma$ increases, the weight of the state $|0,0\rangle$ increases whereas the weight of the state $\left|\chi_{N_{1}}, \mu_{N_{2}}\right\rangle$ decreases. Then the weight of the state $\left|\chi_{N_{1}}, 0\right\rangle$ turns on and it increases with $\Gamma$ until it reaches a maximum value near the $\mathrm{ST}\left(\Gamma \approx \Gamma_{B}^{S T}\right)$. In that case, the superradiant state is fully delocalized over the three states $|0,0\rangle$, $\left|\chi_{N_{1}}, 0\right\rangle$ and $\left|\chi_{N_{1}}, \mu_{N_{2}}\right\rangle$, the corresponding weights being almost identical. As illustrated in Fig. $6 \mathrm{~d}$, when $\Gamma=0$, the subradiant states are superpositions delocalized over the three states $|0,0\rangle,\left|\chi_{N_{1}}, 0\right\rangle$, and $\left|\chi_{N_{1}}, \mu_{N_{2}}\right\rangle$, the weight of the state $\left|\chi_{N_{1}}, 0\right\rangle$ being equal to $0.5 \forall \Gamma$. As $\Gamma$ increases, the weight of the state $|0,0\rangle$ decreases whereas the weight of the state $\left|\chi_{N_{1}}, \mu_{N_{2}}\right\rangle$ increases. Since the weight of the state $|0,0\rangle$ is larger than the weight of the state $\left|\chi_{N_{1}}, \mu_{N_{2}}\right\rangle$ for $\Gamma=0$, it turns out that near ST the weight of these two states is almost identical.

A complementary description of the restructuring of the decaying states is obtained through the characterization of their localized nature. To proceed, let us introduce the inverse participation ratio of a state $\left|\Psi_{i}\right\rangle$ as [55]

$$
\operatorname{IPR}\left(\left|\Psi_{i}\right\rangle\right)=\frac{1}{\sum_{n=1}^{3}\left|\left\langle n \mid \Psi_{i}\right\rangle\right|^{4}} .
$$

Within this definition, a state localized on a site of the trimer is characterized by an IPR equal to 1 . By contrast, the IPR of a state fully delocalized over the trimer is close to 3 .

In that context, the $\Gamma$ dependence of the IPR of the decaying states is shown in Fig. 7 for the configurations A (dashed line) and B (full line). Red curves refer to the subradiant states whereas green curves refer to the superradiant state. For $\Gamma=0$, the extension of the decaying states over the trimer is basically the same whatever the configuration. One obtains $\operatorname{IPR}\left(\left|\Psi_{0}\right\rangle\right)=1.65$ and

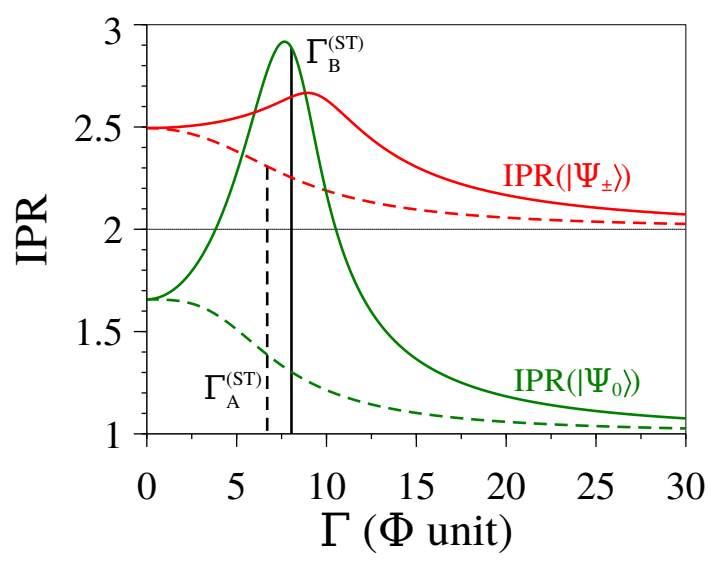

FIG. 7: (Color online) $\Gamma$ dependence of the IPR for configuration A (dashed line) and for configuration B (full line). Red curves refer to the subradiant states and green curves refer to the superradiant state.

$\operatorname{IPR}\left(\left|\Psi_{ \pm}\right\rangle\right)=2.5$. Similarly, for large $\Gamma$ values, the decaying states converge towards asymptotic states whose nature does not depend on the configuration. One obtains $\operatorname{IPR}\left(\left|\Psi_{0}\right\rangle\right)=1$ and $\operatorname{IPR}\left(\left|\Psi_{ \pm}\right\rangle\right)=2$. However, to migrate between these two asymptotic situations, the states follow paths whose nature strongly depends on the configuration. For configuration A, a monotonous transition occurs indicating that the three decaying states loose their extended nature as $\Gamma$ increases. However, for configuration $\mathrm{B}$, a more important state restructuring process takes place. In that case, two peaks appear indicating that both the superradiant and the subradiant states tend to delocalize over all the sites of the trimer. The peak associated to the superradiant state $\left(\operatorname{IPR}\left(\left|\Psi_{0}\right\rangle\right)=\right.$ 2.92) occurs for $\Gamma=7.56 \Phi$ whereas the peak connected to the subradiant states $\left(\operatorname{IPR}\left(\left|\Psi_{ \pm}\right\rangle\right)=2.67\right)$ occurs for $\Gamma=9.00 \Phi$. These peaks clearly arise around the ST transition $\left(\Gamma_{B}^{(S T)}=8.01 \Phi\right)$. At this step, let us mention that theses features have been observed in several simulations carried out for different configurations. For a given set of parameters $\left(N_{1}, N_{2}\right)$, peaks in the IPR appears when $N_{1}>N_{2}$ whereas a monotonous behavior occurs when $N_{2}>N_{1}$.

Finally, the time evolution of specific excitonic populations is shown at the ST in Fig. 7 for the configurations A (Fig. 7a, $\Gamma=\Gamma_{A}^{(S T)}$ ) and B (Fig. $7 \mathrm{~b}, \Gamma=\Gamma_{B}^{(S T)}$ ). For configuration $\mathrm{A}$, as time elapses, the absorbed population $P_{A}(t)$ increases from its initial value equal to zero and it converges to 0.041 in the long time limit, i.e. the value of the initial population of the state $\left|\chi_{N_{1}}, \mu_{N_{2}}\right\rangle$ (blue curve). At the transition, the evolution of $P_{A}(t)$ is modulated by the occurrence of plateaus indicating that the absorbed population exhibits a series of steps during which it remains constant. Such a behavior results from the way the excitonic wave function propagates in the trimer subspace. It can be understood as follows. Initially localized on the third node of the trimer shown on Fig. 1.(d) (i.e. 


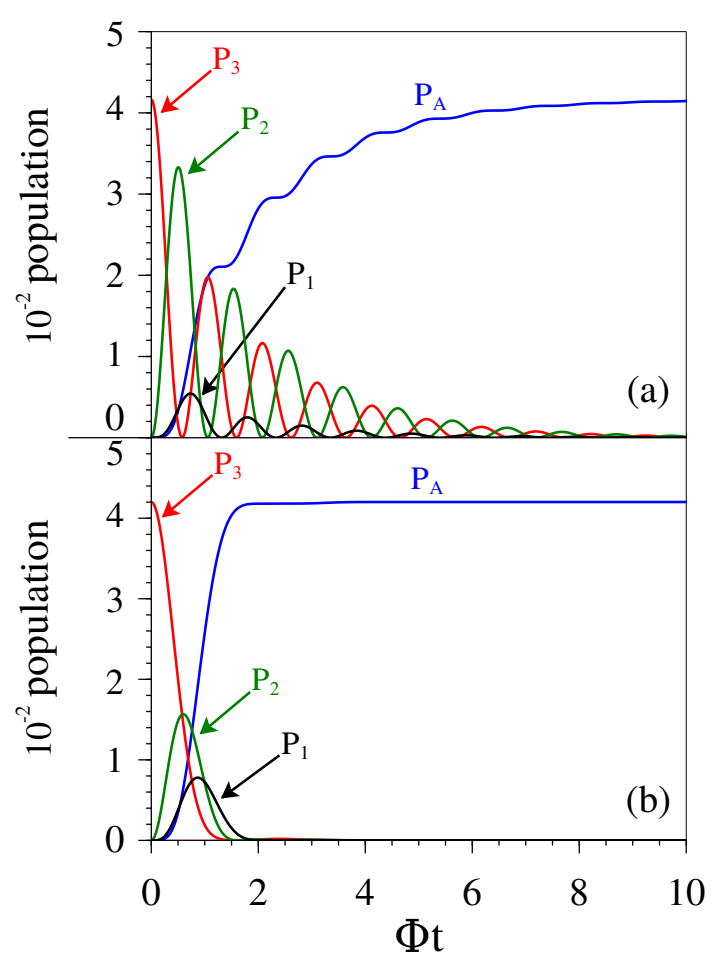

FIG. 8: (Color online) Time evolution of the population at the superradiance transition (a) for configuration A (i.e. for $\Gamma=\Gamma_{A}^{(S T)}$ ), and (b) for configuration B (i.e. for $\left.\Gamma=\Gamma_{B}^{(S T)}\right)$. The figure shows the absorbed population $P_{A}(t)$ (blue curves), the population $P_{3}(t)$ of the state $\left|\chi_{N_{1}}, \mu_{N_{2}}\right\rangle$ (red curves), the population $P_{2}(t)$ of the state $\left|\chi_{N_{1}}, 0\right\rangle$ (green curves) and the population $P_{1}(t)$ of the state $|0,0\rangle$ (black curves) (see the text).

in the state $\left.\left|\chi_{N_{1}}, \mu_{N_{2}}\right\rangle\right)$, the excitonic wave function oscillates between this site and the second site (that is the state $\left.\left|\chi_{N_{1}}, \mu_{N_{2}}\right\rangle\right)$. Therefore, the population of the initial node (red curves) and the population of the central node (green curves) oscillate. Nevertheless, each time the excitonic wave function reaches the central site of the trimer, a small part is transmitted to the first site (i.e. to the state $|0,0\rangle)$. Then, the population of this site turns on and it exhibits damped oscillations (black curve). Each time a part of the excitonic wave function is transmitted to the first site of the trimer, the trap becomes active and the corresponding excitonic population is absorbed. Note that the corresponding absorption time is equal to $\tau=8.80 \Phi^{-1}$.

For configuration B, a fully different behavior arises, as illustrated in Fig. 7b. In that case, a fast absorption takes place. The absorbed population evolves rapidly from zero to $P_{A}(\infty)=0.041$ without exhibiting any plateau (blue curve). Such an efficient transfer results from the dynamics of the excitonic wave function in the trimer subspace. The initial part of the wave function that is localized on the third site of the trimer is directly transferred to the second site. Then, it propagates to the first site where is it absorbed without being able to go back to the other sites. It is as if the exciton wave function behaved as a coherent wave packet insensitive to the dispersion of the trimer. It propagates very quickly to the trap preventing the occurrence of oscillations. An efficient transport arises that give rise to an optimized absorption time equal to $\tau=1.77 \Phi$.

\section{DISCUSSION}

Our numerical results reveal that the exciton dynamics is governed by two kinds of eigenstates. First, the exciton Hamiltonian supports $N_{1}\left(N_{2}+1\right)-2$ eigenstates associated to real eigenvalues. These states, whose degeneracy is very pronounced, are insensitive to the presence of the trap. Then, the exciton supports three decaying eigenstates which are those of the restriction of the Hamiltonian to the trimer subspace generated by the three states : $|0,0\rangle,\left|\chi_{N_{1}}, 0\right\rangle$ and $\left|\chi_{N_{1}}, \mu_{N_{2}}\right\rangle$. Characterized by complex energies, i.e. finite lifetimes, the three decaying states are the only states that contribute to the trapping process.

In that context, it has been shown that the excitonic population absorbed by the trap depends on the size parameters, only. The larger the size is, the smaller is the absorbed population. This absorbed population is invariant under the exchange of the parameters $N_{1}$ and $N_{2}$ and it is independent of the absorption rate $\Gamma$. By contrast, all the parameters $N_{1}, N_{2}$ and $\Gamma$ control the dynamics of the trapping, i.e. the time required to absorb the excitonic population. When theses parameters are judiciously chosen, the efficiency of the transfer is optimized resulting in the minimization of the absorption time. The analysis of the system eigenstates reveals that such a feature arises around the ST.

Indeed, the decaying states are divided into two kinds of states : one superradiant state and two subradiant states. The width of the superradiant state increases monotonously as $\Gamma$ increases whereas the width of the subradiant states behaves non monotonously. The latter reaches a maximum value for a critical value of $\Gamma$ which is the signature of the ST. We have shown that the transition is accompanied by a restructuring process of the decaying states that strongly depends on the size parameters $N_{1}$ and $N_{2}$. Consequently, for a same total absorbed population, two situations have been brought to light where the transport efficiency is either superoptimized if $N_{1}>N_{2}$, or sub-optimized if $N_{2}>N_{1}$.

To interpret these features, let us mention that the quantum dynamics that emerges when the exciton is initially located on a peripheral site of the graph exhibits two contributions (see Eq.(9)). First, a part of the initial wave function is projected on the decaying states. These states exhibiting finite lifetimes, this contribution tends to zero in the long time limit. The second contribution results from the projection of the initial wave function on the remaining degenerate eigenstates. These states be- 
ing stable as time elapses, they favor a coherent exciton dynamics during which the corresponding part of the exciton population is conserved. However, as mentioned in Sec. III.A, the degeneracy is responsible for a localization mechanism so that the conserved excitonic population is mainly located on the excited star. In that context, in the long time limit, the unconserved part of the excitonic population disappears so that the survival probability is given by the probability to observe the exciton in the stable eigenstates $\left|\chi_{k}, \mu_{q}\right\rangle$ and $\left|\chi_{k}, \pm\right\rangle$. After straightforward calculations, one obtains $Q(\infty)=1-1 / N_{1} N_{2}$, so that the absorbed population is

$$
P_{A}(\infty)=\frac{1}{N_{1} N_{2}} .
$$

In a perfect agreement with what was observed in Fig. $3, P_{A}(\infty)$ depends on the same way on the two size parameters. Moreover, this quantity is always larger than or equal to the maximum value $P_{0,0 \mid \ell_{0}, s_{0}}^{\max }=4 /\left(N_{1}+N_{2}\right)^{2}$ of the population that reaches the central node without the trap (see Sec. III.A). In other words, the presence of the trap improves the transfer from the periphery to the core of the graph. Note that the two populations are equal when $N_{1}=N_{2}$.

Let us now discuss the influence of the parameters on the optimization of the transport efficiency, specifically on the minimization of the absorption time. This phenomenon originates in the evolution of the structure of the decaying states between two asymptotic vectors, as $\Gamma$ increases from zero to infinity. Indeed, when $\Gamma=0$, the superradiant state is a superposition involving the two states $|0,0\rangle$ and $\left|\chi_{N_{1}}, \mu_{N_{2}}\right\rangle$ whereas the subradiant states are decomposed over the three states $|0,0\rangle,\left|\chi_{N_{1}}, 0\right\rangle$ and $\left|\chi_{N_{1}}, \mu_{N_{2}}\right\rangle$ (see Eq.(8)). In a marked contrast, for large $\Gamma$ values, the superradiant state localizes on the core of the graph and tends to $|0,0\rangle$. It is characterized by a width equal to $\Gamma$. In turn, the subradiant states tend to the superpositions $\left(\left|\chi_{N_{1}}, 0\right\rangle \pm\left|\chi_{N_{1}}, \mu_{N_{2}}\right\rangle\right) / \sqrt{2}$ and they become insensitive to the trap.

In that context, the way the parameter $\Gamma$ controls the optimization of the transport efficiency can be understood as follows. When $\Gamma$ increases from zero, the width of the three decaying states increases. For small $\Gamma$ values, the three widths are of the same order of magnitude so that the three decaying states participate in the absorption process over a similar timescale. The optimization is thus obtained when the absorption time $\tau$ is minimized, that is when the average width of the decaying states is maximized. This phenomenon occurs at the ST $\left(\Gamma \approx \Gamma^{(S T)}\right)$. Beyond the transition, the width of the superradiant state still increases with $\Gamma$ whereas the width of the subradiant states decreases. Consequently, the average width of the decaying states decreases and one deviates from the region of the parameter space where the optimization takes place. In addition, this deviation is enhanced by the fact that the superradiant state localizes on the core of the graph and it no longer allows the transfer from the periphery to the core.
But the absorption rate $\Gamma$ is not the only parameter that influence the optimization process. Indeed, we have observed that the size parameters $N_{1}$ and $N_{2}$ play a crucial role, especially in the restructuring of the states that arises at the ST. This feature can be understood by considering the two asymptotic situations $N_{1} \ll N_{2}$ and $N_{1} \gg N_{2}$.

When $N_{1} \ll N_{2}$, the restriction of the Hamiltonian to the trimer subspace corresponds basically to an isolated state $|0,0\rangle$ decoupled form the two remaining states $\left|\chi_{N_{1}}, 0\right\rangle$ and $\left|\chi_{N_{1}}, \mu_{N_{2}}\right\rangle$ (see Fig. 1d). Consequently, for small $\Gamma$ values, the superradiant and subradiant states look like the asymptotic states to which they must tend for large $\Gamma$ values. These asymptotic states, because of their structure, prevent the occurrence of an efficient transfer between the periphery and the core of the graph. Therefore, as $\Gamma$ increases, the transport efficiency is optimized through the maximization of the widths at the decaying states. But this transition is not accompanied by a major restructuring of that states. We can speak of a sub-optimization induced by the ST.

Conversely, when $N_{1} \gg N_{2}$, the restriction of the Hamiltonian to the trimer subspace corresponds now to an isolated state $\left|\chi_{N_{1}}, \mu_{N_{2}}\right\rangle$ decoupled form the two remaining states $|0,0\rangle$ and $\left|\chi_{N_{1}}, 0\right\rangle$ (see Fig. 1d). Therefore, for small $\Gamma$ values, the superradiant and subradiant states are fundamentally different from the asymptotic states to which they must tend for large $\Gamma$ values. Consequently, the maximization of the width of the decaying states at the superradiance transition is accompanied by a major state restructuring. Such a restructuring results in the occurrence of states that are almost fully delocalized over the three trimer states $|0,0\rangle,\left|\chi_{N_{1}}, 0\right\rangle$ and $\left|\chi_{N_{1}}, \mu_{N_{2}}\right\rangle$, thus promoting a very efficient transfer between the periphery and the core of the graph. In other words, the maximization of the widths combined with the important restructuring of the states favor a superoptimization of the transport efficiency.

Of course, when $N_{1}$ and $N_{2}$ are quite similar, an intermediate situation arises. The maximization of the width of the decaying states at the superradiance transition is now accompanied by a moderate state restructuring. The optimization regime of the transport efficiency lies somewhere between the super-optimization and the suboptimization. This feature is illustrated in Fig. 9 that shows the size dependence of the optimized value of the absorption time $\tau$. The case $\left(N_{1}=10, N_{2}=3\right)$ specifies the super-optimized regime where the minimum value of the absorption time reduces to $1.68 \Phi^{-1}$. This absorption time increases as one moves towards the sub-optimized regime where it reaches $9.84 \Phi^{-1}$ for $\left(N_{1}=3, N_{2}=10\right)$. At the border between the two regimes, i.e. for $N_{1}=$ $N_{2}$, the minimized absorption time is typically of about $3.5 \Phi^{-1}$. 


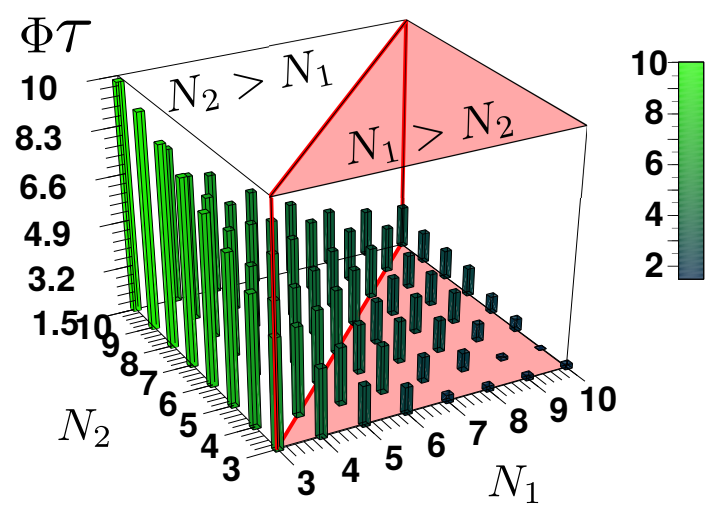

FIG. 9: (Color online) Size dependence of the minimum value of the absorption time $\tau$.

\section{CONCLUSION}

In this paper, a tight-binding model was introduced for describing the dynamics of an exciton moving on an extended star graph whose central node is occupied by a trap. On this graph, the exciton dynamics is governed by two kinds of eigenstates. First, the exciton exhibits many eigenstates associated to real eigenvalues and whose degeneracy is very pronounced. Then, the exciton supports three decaying eigenstates characterized by complex energies, i.e. finite lifetimes. Consequently, the quantum dynamics that emerges when the exciton is initially located on a peripheral site of the graph exhibits two distinct contributions. First, the part of the initial wave function projected on the degenerate eigenstates favors a coherent dynamics during which the corresponding part of the exciton population is conserved. Owing to the degeneracy, this conserved population remains confined over the few sites that surround the excited site. The second contribution results from the projection of the initial wave function on the three decaying states. These states exhibiting finite lifetimes, that are the only states that contribute to the trapping process.

In that context, it has been shown that the excitonic population absorbed by the trap depends on the size of the graph, only. By contrast, both the size parameters and the absorption rate control the value of the time needed to absorb the excitonic population. When theses parameters are judiciously chosen, the efficiency of the transfer is optimized resulting in the minimization of the absorption time. The analysis of the system eigenstates reveals that such a feature arises around the so-called superradiance transition for which the average width of the decaying states is maximized. Nevertheless, we have shown that the transition is accompanied by a restructuring of the decaying states that strongly depends on the size parameters. Depending on these parameters, two situations have been highlighted where the transport efficiency is either super-optimized or sub-optimized, for the same value of the total absorbed population.

In the present work, superradiance-induced optimization of the transport efficiency was investigated in a quite simple graph. Although interesting features have been observed, additional studies are required to investigate what happens in more intricate situations. For instance, it could be wise to consider more complex graphs such as dendrimers as well as the presence of disorder inherent to realistic systems. Moreover, from a physical point of view, the exciton does not propagate freely but it interacts with its surrounding that favors energy relaxation and/or dephasing. These effects must be included in our formalism to establish a more general description of the trapping phenomena at finite temperature.

\section{APPENDIX A: PROBABILITIES}

When $\Gamma=0$, the Hamiltonian can be diagonalized exactly. As a consequence, it is straightforward to calculate the exciton density whose analytical expression is defined as

$$
\begin{aligned}
P_{\ell_{0}, s_{0} \mid \ell_{0}, s_{0}}(t) & =\left|\frac{1+\left(N_{1}+N_{2}\right)\left(N_{2}-1\right)}{N_{2}\left(N_{1}+N_{2}\right)}+\frac{N_{1}-1}{N_{2} N_{1}} \cos \left(\sqrt{N_{2}} \Phi t\right)+\frac{\cos \left(\sqrt{N_{1}+N_{2}} \Phi t\right)}{N_{1}\left(N_{1}+N_{2}\right)}\right|^{2} \\
P_{\ell_{0}, 0 \mid \ell_{0}, s_{0}}(t) & =\left|\frac{N_{1}-1}{N_{1} \sqrt{N_{2}}} \sin \left(\sqrt{N_{2}} \Phi t\right)+\frac{\sin \left(\sqrt{N_{1}+N_{2}} \Phi t\right)}{N_{1} \sqrt{N_{1}+N_{2}}}\right|^{2} \\
P_{\ell_{0}, s \neq s_{0} \mid \ell_{0}, s_{0}}(t) & =\left|\frac{N_{1}-1}{N_{1} N_{2}} \cos \left(\sqrt{N_{2}} \Phi t\right)+\frac{1-N_{1}-N_{2}}{N_{2}\left(N_{1}+N_{2}\right)}+\frac{\cos \left(\sqrt{N_{1}+N_{2}} \Phi t\right)}{N_{1}\left(N_{1}+N_{2}\right)}\right|^{2} \\
P_{0,0 \mid \ell_{0}, s_{0}}(t) & =\left|\frac{\cos \left(\sqrt{N_{1}+N_{2}} \Phi t\right)-1}{N_{1}+N_{2}}\right|^{2} \\
P_{\ell \neq \ell_{0}, 0 \mid \ell_{0}, s_{0}}(t) & =\left|\frac{1}{N_{1} \sqrt{N_{2}}} \sin \left(\sqrt{N_{2}} \Phi t\right)-\frac{\sin \left(\sqrt{N_{1}+N_{2}} \Phi t\right)}{N_{1} \sqrt{N_{1}+N_{2}}}\right|^{2} \\
P_{\ell \neq \ell_{0}, s \neq 0 \mid \ell_{0}, s_{0}}(t) & =\left|\frac{1}{N_{2}\left(N_{1}+N_{2}\right)}+\frac{\cos \left(\sqrt{N_{1}+N_{2}} \Phi t\right)}{N_{1}\left(N_{1}+N_{2}\right)}-\frac{1}{N_{1} N_{2}} \cos \left(\sqrt{N_{2}} \Phi t\right)\right|^{2}
\end{aligned}
$$


The corresponding limiting probabilities are expressed as

$$
\begin{aligned}
\bar{P}_{\ell_{0}, s_{0} \mid \ell_{0}, s_{0}} & =\frac{1}{2\left(N_{1} N_{2}\right)^{2}}\left(\frac{2 N_{1}^{2}+N_{2}^{2}}{\left(N_{1}+N_{2}\right)^{2}}+\frac{4 N_{1}^{2}\left(N_{2}-1\right)}{N_{1}+N_{2}}+2 N_{1}^{2}\left(N_{2}-1\right)^{2}+\left(N_{1}-1\right)^{2}\right) \\
\bar{P}_{\ell_{0}, 0 \mid \ell_{0}, s_{0}} & =\frac{1}{2\left(N_{1} N_{2}\right)^{2}}\left(\frac{N_{2}^{2}}{N_{1}+N_{2}}+N_{2}\left(N_{1}-1\right)^{2}\right) \\
\bar{P}_{\ell_{0}, s \neq s_{0} \mid \ell_{0}, s_{0}} & =\frac{1}{2\left(N_{1} N_{2}\right)^{2}}\left(\frac{2 N_{1}^{2}+N_{2}^{2}}{\left(N_{1}+N_{2}\right)^{2}}-\frac{4 N_{1}^{2}}{N_{1}+N_{2}}+2 N_{1}^{2}+\left(N_{1}-1\right)^{2}\right) \\
\bar{P}_{0,0 \mid \ell_{0}, s_{0}} & =\frac{3}{2\left(N_{1}+N_{2}\right)^{2}} \\
\bar{P}_{\ell \neq \ell_{0}, 0 \mid \ell_{0}, s_{0}} & =\frac{1}{2\left(N_{1} N_{2}\right)^{2}}\left(\frac{N_{2}^{2}}{N_{1}+N_{2}}+N_{2}\right) \\
\bar{P}_{\ell \neq \ell_{0}, s \neq 0 \mid \ell_{0}, s_{0}} & =\frac{1}{2\left(N_{1} N_{2}\right)^{2}}\left(1+\frac{2 N_{1}^{2}+N_{2}^{2}}{\left(N_{1}+N_{2}\right)^{2}}\right)
\end{aligned}
$$

[1] V. May and O. Kuhn, Charge and Energy Transfer Dynamics in Molecular Systems (Wiley-VCH Verlag, Berlin, 2000).

[2] T. Renger, V. May, and Oliver Kuhn, Phys. Rep. 343, 137 (2001).

[3] J. Knoester and V.M. Agranovich, Thin Films and Nanostructures: Electronic Excitations in Organic Based Nanostructures (Elsevier, Amsterdam, 2003).

[4] A.C. Scott, Phys. Rep. 217, 1 (1992).

[5] V. Pouthier, J.C. Light, and C. Girardet, J. Chem. Phys. 114, 4955 (2001).

[6] R.M. Pearlstein, J Chem. Phys. 56, 2431 (1972).

[7] D.L. Huber, Phys. Rev. B 20, 2307 (1979).

[8] D.L. Huber, Phys. Rev. B 22, 1714 (1980).

[9] P.E. Parris, Phys. Rev. Lett. 62, 1392 (1989).

[10] V.A. Malyshev, A. Rodriguez, and F. DominguezAdame, J. Lumin. 81, 127 (1999).

[11] D.L. Huber, Phys. Rev. B 45, 8947 (1992).

[12] R.S. Grinyov, A.V. Sorokin, G. ya. Gural'chuk, S.L. Efimova, I.A. Borovoi, and Yu.V. Malyukin, Theor. Exp. Chem. 45, 58 (2009).

[13] V.M. Kenkre and Y.M. Wong, Phys. Rev. B 23, 3748 (1981).

[14] J. A. Tuszynski, M. F. Jorgensen, and D. Mobius, Phys. Rev. E 59, 4374 (1999).

[15] R.P. Hemenger, K. Lakatos-Lindenberg, and R.M. Pearlstein, J. Chem. Phys. 60, 3271 (1974).

[16] D. Astruc, E. Boisselier, and C. Ornelas, Chem. Rev. 110, 1857 (2010).

[17] R.E. Fenna and B.W. Matthews, Nature 258, 573 (1975).

[18] K. Harigaya, Chem. Phys. Lett. 300, 33 (1999).

[19] M.A. Martin-Delgado, J. Rodriguez-Laguna, and G. Sierra, Phys. Rev. B 65, 155116 (2002).

[20] C. Supritz, A. Engelmann, and P. Reineker, J. Lumin. 111, 367 (2005).

[21] S. Tretiak, V. Chernyak, and S. Mukamel, J. Phys. Chem. B 102, 3310 (1998).

[22] M. Nakano, M. Takahata, H. Fujita, S. Kiribayashi, and K. Yamaguchi, Chem. Phys. Lett. 323, 249 (2000).
[23] G.W. Crabtree and N.S. Lewis, Phys. Today 60, 37 (2007).

[24] A. Bar-Haim, J. Klafter, and R. Kopelman, J. Am. Chem. Soc. 119, 6197 (1997).

[25] M.S. Choi, T. Aida, T. Yamazaki, and I. Yamazaki, Chem. Eur. J. 8, 2667 (2002).

[26] M.B. Plenio and S.F. Huelga, New. J. Phys. 10, 113019 (2008).

[27] M. Mohseni, P. Rebentrost, S. Lloyd, and A. AspuruGuzik, J. Chem. Phys. 129, 174106 (2008).

[28] P. Rebentrost, M. Mohseni, I. Kassal, S. Lloyd, and A. Aspuru-Guzik, New. J. Phys. 11, 033003 (2009).

[29] J. Wu, F. Liu, Y. Shen, J. Cao and R.J. Silbey, New. J. Phys. 12, 105012 (2010).

[30] J. Wu, F. Liu, J. Ma, R.J. Silbey, and J. Cao, J. Chem. Phys. 137, 174111 (2012).

[31] O. Mulken and A. Blumen, Phys. Rep. 502, 37 (2011).

[32] A.M. Childs, Phys. Rev. Lett. 102, 180501 (2009).

[33] S.E. Venegas-Andraca, Quantum Inf. Process. 11, 1015 (2012).

[34] A. Volta, J. Phys. A: Math. Theor. 42, 225003 (2009).

[35] Z. Darazs, A. Anishchenko,T. Kiss, A. Blumen, and O. Mulken, Phys. Rev. E 90, 032113 (2014).

[36] X.P. Xu, Phys. Rev. E 79, 011117 (2009).

[37] P.L. Krapivsky, J.M. Luck, and K. Mallick, J. Stat. Phys. 154, 1430 (2014).

[38] E. Agliari, O. Mulken, and A. Blumen, Int. J. Bifurcation Chaos 20, 271 (2010).

[39] O. Mulken, A. Blumen, T. Amthor, C. Giese, M. ReetzLamour, and M. Weidemuller, Phys. Rev. Lett. 99, 090601 (2007).

[40] O. Mulken and A. Blumen, Physica E 42, 576 (2010).

[41] V. V. Sokolov and V. G. Zelevinsky, Nucl. Phys. A 504, 562 (1989).

[42] V. V. Sokolov and V. G. Zelevinsky, Ann. Phys. (N.Y.) 216, 323 (1992).

[43] G.L. Celardo and L. Kaplan, Phys. Rev. B 79, 155108 (2009).

[44] R.H. Dicke, Phys. Rev. 93, 99 (1954). 
[45] V.V Sokolov and V.G. Zelevinsky, Ann. Phys. (NY) 216, 323 (1992).

[46] V.V Sokolov and V.G. Zelevinsky, Nucl. Phys. A 504, 562 (1989).

[47] G.L. Celardo, F. Borgonovi, M. Erkli, V.I. Tsifrinovich, and G.P. Berman, J. Phys. Chem.C 116, 22105 (2012).

[48] G. G. Giusteri, G.L.Celardo, and F. Borgonovi, Phys. Rev. E 93, 032136 (2016).

[49] Y. Zhang, G.L.Celardo, F. Borgonovi, and L. Kaplan, Phys. Rev. E 95, 022122 (2017).

[50] A. Sequeira, Interconnecting Cisco Network Devices Part I (ICND1) : Foundation Learning Guide (Cisco Press, Indianapolis, 2013).
[51] D.C. Brody, J. Phys. A: Math. Theor. 47, 035305 (2014).

[52] D. Aharonov, A. Ambainis, J. Kempe, and U. Vazirani, in Proceedings of the thirty-third annual ACM symposium on Theory of computing (STOC01) (ACM Press, New York, 2001), p.50.

[53] O. Mulken and A. Blumen, Phys. Rev. E 73, 066117 (2006).

[54] O. Mulken, V. Bierbaum, and A. Blumen, J. Chem. Phys. 124, 124905 (2006).

[55] B. Kramer and A. MacKinnon, Rep. Prog. Phys. 56, 1469 (1993). 